\title{
Monitoring of Physical Activity of Soldiers of the Army of the Czech Republic
}

\author{
Jan Zemánek, Miroslava Přidalová \\ Palacký University Olomouc, Faculty of Physical Culture, CZECH REPUBLIC
}

\begin{abstract}
Aim: This research is aimed at the possibilities of physical training of Army of the Czech Republic personnel (soldiers of the $7^{\text {th }}$ mechanised brigade) during a routine week spent in garrison and the follow-up analysis of the amount of their physical activity.

Methodology: The observed group was made up of 52 professional soldier volunteers in the age range of $29.9 \pm 4.5$ years. The measurement has been performed continuously during the time span of one week. Physical activity has been measured with the utilization of the ActiGraph GT1M accelerometers supplemented with the use of a weekly physical activity protocol.

Results: Based on the results it is possible to infer that the average daily physical activity (7.66 hours) has been greater than the average daily physical inactivity (5.89 hours). No difference in the amount of weekly physical activity of professional soldiers in relation to age ( $\leq 30$ years and $>30$ years) has been found, nor in relation to being a smoker/non-smoker, nor in relation to $B M I\left(<24.9 \mathrm{~kg} / \mathrm{m}^{2}\right.$ and $\geq$ $\left.24.9 \mathrm{~kg} / \mathrm{m}^{2}\right)$.

Conclusion: With an average daily step count of 10169.84 the amount of physical activity of the soldiers is sufficient and meets the health recommendation for the daily step count (Aoyagi \& Shephard, 2009).
\end{abstract}

Key words: ActiGraph, motion, physical activity, BMI, monitoring.

\section{INTRODUCTION}

There are multiple types of professional specialisation of the personnel in the military environment. The most basic division is the split between personnel of combat units and of supporting units, alternatively staff personnel. Physical training is conducted with dissimilar intensity and objectives on different organisational levels as there is usually a higher level of physical fitness requirements for combat units in comparison to other units. Apart from their expert and psychological preparedness, physical fitness is especially important for military personnel to successfully manage set tasks, functions and situations related to military life. Physical fitness is the objective of physical training which is aimed at providing physical fitness of the soldiers via a pedagogically controlled process to secure the ability of the soldiers to properly perform the tasks arising from their assigned military functions (Ministry of Defence, 2011). The amount of training hours spent on different types of training - basic ability and skill development, obstacle course, endurance development, self-defence techniques, swimming, sport games - is firmly set in military regulations (Prog-1-3, 2005).

Good physical fitness of Army personnel is considered a necessary requirement for the effective performance of their duties, be it during ordinary activities in garrison (specialist training, guard duty, vehicle maintenance and repair, etc.), during continuous field training and exercises, during several months long military operations abroad, during nature catastrophe relief and humanitarian tasks, tasks in assistance of the Police of the Czech Republic as part of the Integrated Rescue System or during public presentations of military capabilities - combat training presentations, sporting events and others (Zemánek, 2015). 
Knowledge of the current state of a soldier's physical fitness is regarded as a necessary requirement for effective job assignment of professional soldiers (it is also a necessary requirement for constructing an appropriate training programme) and data about its development represent a valuable feedback information in relation to the quality of the training.

\section{METHODOLOGY}

The observed set was made up of 52 professional soldier volunteers, members of the 73th tank battalion, 7th mechanized brigade. Measurement of weekly physical activity was based on voluntariness and has been conducted with consent of the participants. The measurement has been conducted during an ordinary week (Mon-Sun) spent in the garrison. 52 participants (male) of the age of $29.9 \pm 4.5$ years took part in the monitoring. The weather during the week (in April) has been sunny to partly cloudy with temperatures during the day 11-16 degree of Celsius. Working hours of soldiers are 40 hours a week (Monday-Thursday from 7:00 AM to 4:00 PM, Friday from 7:00 AM to 1:30 PM, free weekends). Somatic parameters of the monitored group are in Table 1.

Tab. 1: Somatic parameters of the monitored group

\begin{tabular}{|l|l|c|c|c|c|}
\hline Nr. & Variable & M & SD & Min & Max \\
\hline 1 & Weight $[\mathrm{kg}]$ & 81.8 & 9.6 & 64.0 & 110.0 \\
\hline 2 & Height $[\mathrm{cm}]$ & 179.4 & 7.1 & 161.0 & 193.0 \\
\hline 3 & Age [years] & 29.9 & 4.5 & 22.3 & 42.5 \\
\hline 4 & BMI $\left[\mathrm{kg} / \mathrm{m}^{2}\right]$ & 25.5 & 3.1 & 19.4 & 32.1 \\
\hline
\end{tabular}

The average BMI value of the group is 25.5. According to Vitek (2008) this value corresponds to the overweight category, but for $80 \%$ of the group, who had a BMI value greater than $24.9 \mathrm{~kg} / \mathrm{m}^{2}$, this was caused by a higher ratio of muscle mass resulting in a higher body weight and thus in a higher BMI value. None of the group had a BMI value smaller than $18.5 \mathrm{~kg} / \mathrm{m}^{2}$, which would correspond to malnutrition.

In accordance with the Military Personnel Law (Law no. 221/1999 Collection) it is necessary to consider physical fitness a basic obligation of a professional soldier. Physical training is a controlled physical education activity in accordance with internal normative acts and is obligatory for all military personnel fit for duty. The aim of physical training is to secure the ability of the soldiers to properly perform the tasks arising from their assigned military functions via a pedagogically controlled process. It is realised via practical training which is being conducted based on an approved training schedule in accordance with training programmes and other training regulations. It is being methodically directed by the Physical Training Chief of the Ministry of Defence via physical training specialists or via personnel designated to direct physical training. The scheme of physical training is shown in Figure 1 (Ministry of Defence, 2011). 


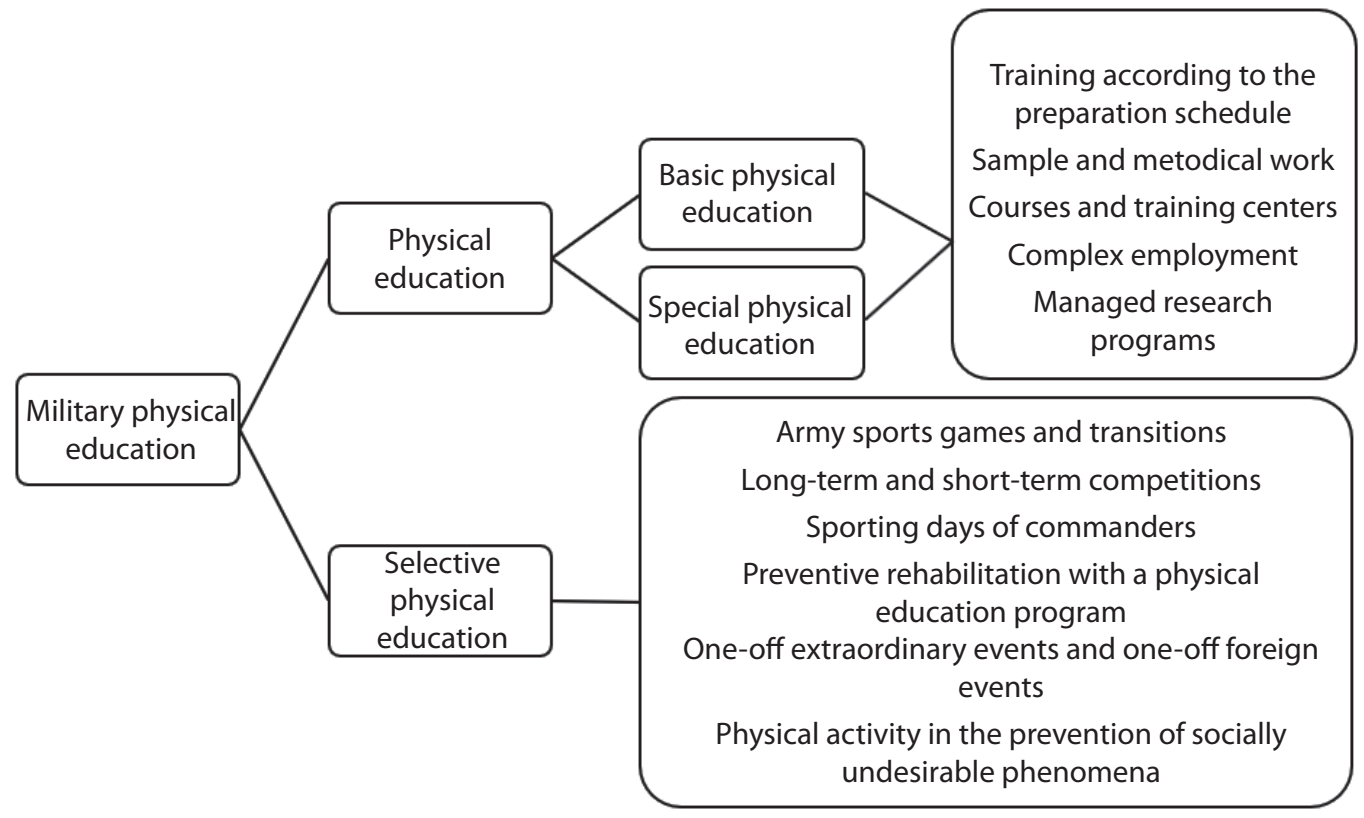

Fig. 1: Scheme of physical training (Ministry of Defence, 2011)

Physical training is one the main types of military training. Its aim is to provide physical fitness of military personnel in order to manage their duties and strain during all situations.

To provide for the needs of organised and individual physical training of the personnel of the 73th tank battalion there is a 333 metres long running course available in the garrison and paved and unpaved running courses in the vicinity of the garrison (lengths of 1500 metres to 20 kilometres). A collective activity of the 73th tank battalion is the battalion run which is being organised once per week. Its main objective is the increase of individual physical fitness and the increase of collective cohesiveness of the battalion personnel. During unfavourable climatic conditions $(<-15$ degree of Celsius) the battalion run is substituted by the battalion march (with or without load). The objective of the march is the development of individual physical and psychological endurance of battalion personnel. Another means used for physical fitness development is the NATO obstacle course, located in the 15 kilometres distant Vyškov garrison. 15 kilometres distant from the Pŕáslavice garrison, in MTA (military training area) Libavá, a combat obstacle course is located, offering multiple technical sections - for example water surface crossings using a rope, two-storeyed building rope climbing with a subsequent down-jump, movement in restricted space and others. The Přáslavice garrison itself also offers a climbing facility.

Monitoring of physical activity has been conducted using the uniaxial accelerometer ActiGraph GT1M, which has provided continuous week-long monitoring of physical activity. The accelerometer ActiGraph GT1M measures the frequency, time and intensity of motion and provides an objective monitoring of the physical activity of the volunteers without age restriction.

Based on the time the device has been worn and the measured physical activity it is possible to calculate also the physical inactivity of the volunteers, which represents a state of the organism with minimal body movement with energy requirements approximately equal to those of basal metabolism. We get the average active energy output performance as the ration of the active energy output and the time during which the accelerometer has been measuring the motion activity. Despite its simple construction, the accelerometer is considered a valid and dependable device for motion activity monitoring, particularly during multiple day or weekly-long use (Aadland et al., 2015; Abel et al., 2008; Rohtney et al., 2008). It has no negative effects on the wearer's health 
and does not produce any kind of EM radiation (Mitáš et al., 2007). The device has always been worn during the whole day, with the exception of showering, bathing and swimming since it is not waterproof. The ActiGraphGT1M is storing average data over minute long intervals with the possibility to ascertain how many minutes have been spent with light, average or intensive physical activity during days and weeks (Mitáš et al., 2007).

Uniaxial accelerometers like the ActiGraph provide acceleration measurement only in the vertical plane resulting in a possible lower validity of the measurement during higher velocities of running locomotion in the sense of underestimating the energetic output (Brage et al., 2003). The device is able to monitor and store temporary data on the activity in relatively short time intervals for the time of days up to weeks (Montoye et al., 1996). Accelerometer ActiGraph GT1M is shown in Figure 2. Figure 3 shows the correct way of wearing the device.

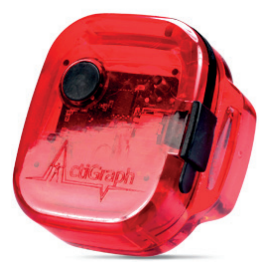

Fig. 2: ActiGraph GT1M (Mitáš et al., 2007)

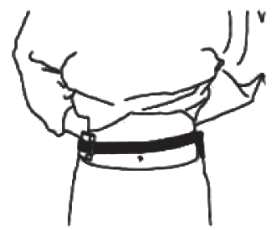

Fig. 3: Correct wearing of the ActiGraph GT1M (Mitáš et al., 2007)

The measured data has been processed using the ActiPA2006 software developed for research purposes of the Centre of Kinantropology Research of the Faculty of Physical Culture, Palacký University, Olomouc (Chytil, 2006). All statistical calculations have been made with STATISTICA 8.0. The descriptive characteristic has been expressed as medians, arithmetic averages, interquartile ranges and maximal and minimal values.

The t-test and Kruskal-Wallis test have been used for inter-group variable comparison. Statistical significance has been determined at a level of $p<0.05$. 


\section{RESULTS}

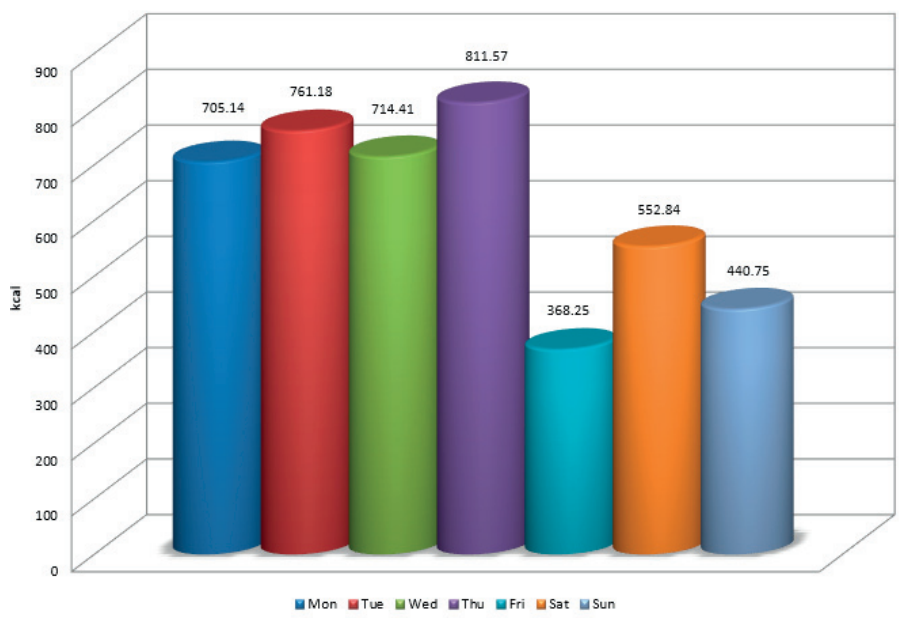

Fig. 3: Active energy output [kcal] during days of the week

Figure 3 shows the values of active energy output [kcal] during separate days of the week, which have been approximately at the same level during the week with the exception of Fridays when the output has been at its minimum. This corresponds to the average step count during this day, when this value has also been minimal.

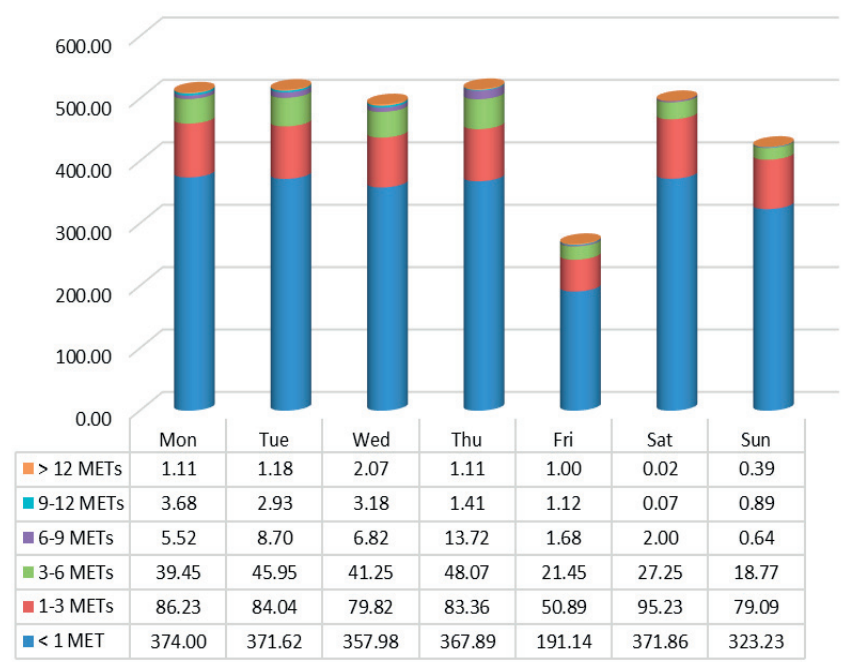

Fig. 4: Structure of physical activity [min] during the week based on average intensity of physical [MET]

Figure 4 shows the structure of physical activity in separate days during the week and during the whole week. The structure is given by the time duration [min] of different intensity of physical activity, from activity intensity $<1$ MET to $>12$ METs. 


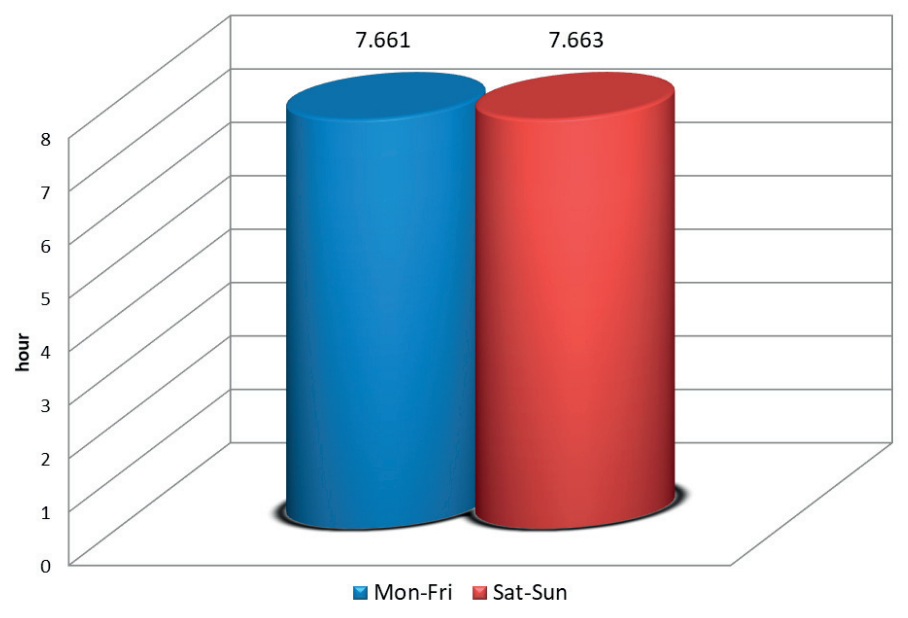

Fig. 5: Average physical activity [hours]

The graph shown in Figure 5 implies a higher average physical activity during the weekend, but the difference is not large enough to be statistically significant $(p=0.997)$.

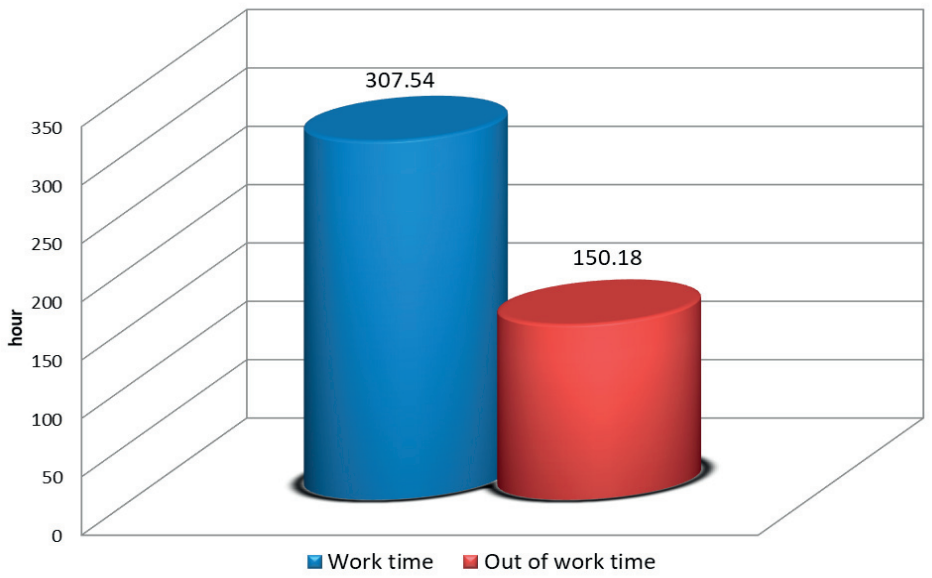

Fig. 6: Average physical activity [min] during working hours vs off-duty time

The average physical activity [min] during working hours has been more than double the average physical activity during off-duty time as shown in Figure 6 . The difference is statistically significant $(\mathrm{p}=0.000)$. 


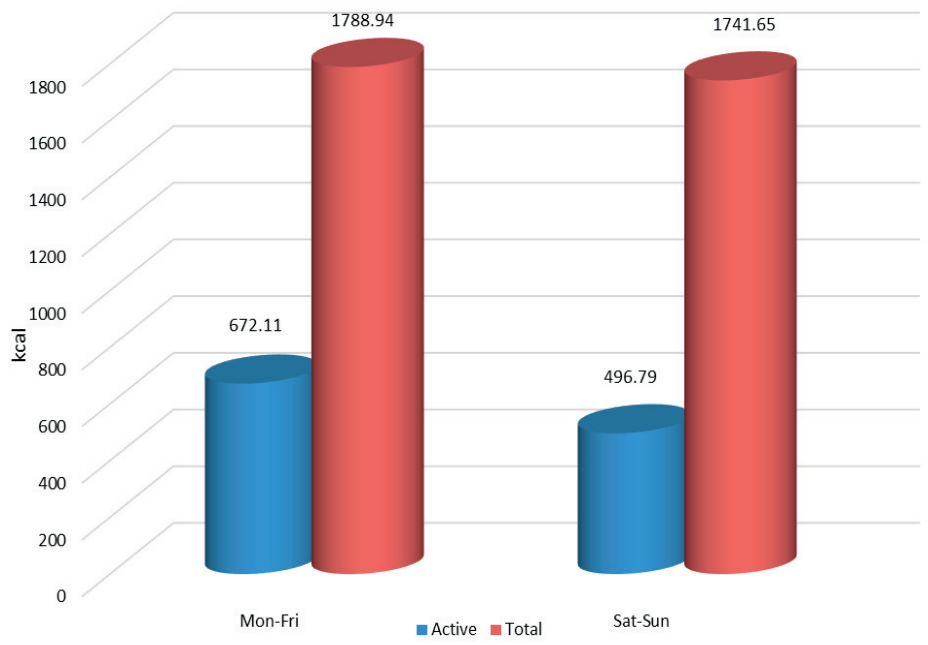

Fig. 7: Average energy output [kcal]

The graph shown in Figure 7 implies that the average active energy output value [kcal] calculated for one day, as well as the overall energy output [kcal] calculated for one day, was higher during the weekdays than during the weekend.

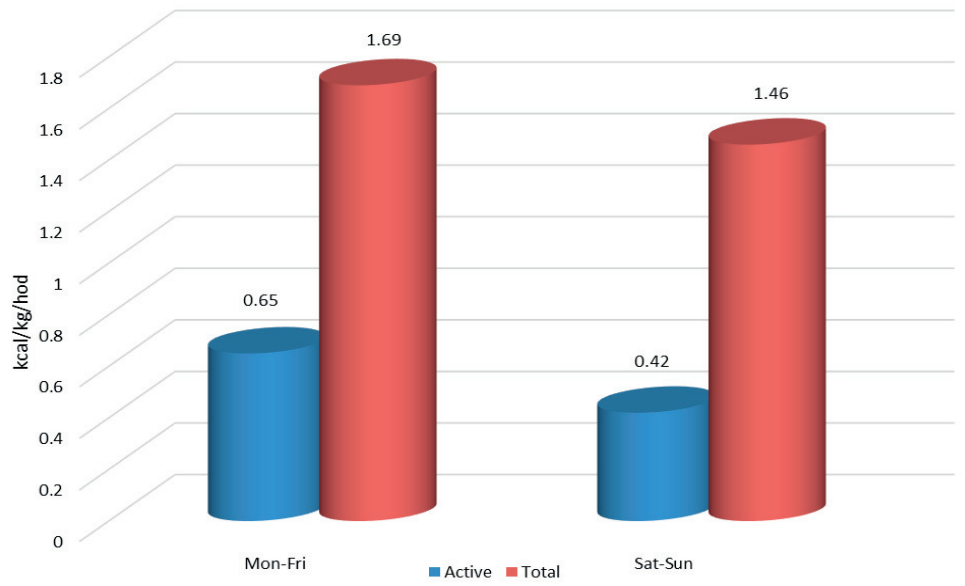

Fig. 8: Average relative output [kcal/kg/hour]

The difference between weekdays and weekends is evident from the graph shown in Figure 8 for these values:

Average active relative output [ $\mathrm{kcal} / \mathrm{kg} / \mathrm{hour}]$ calculated for one day and overall relative output calculated for one day. These were higher on weekdays than on the weekends with the difference being statistically significant $(\mathrm{p}=0.000)$. 


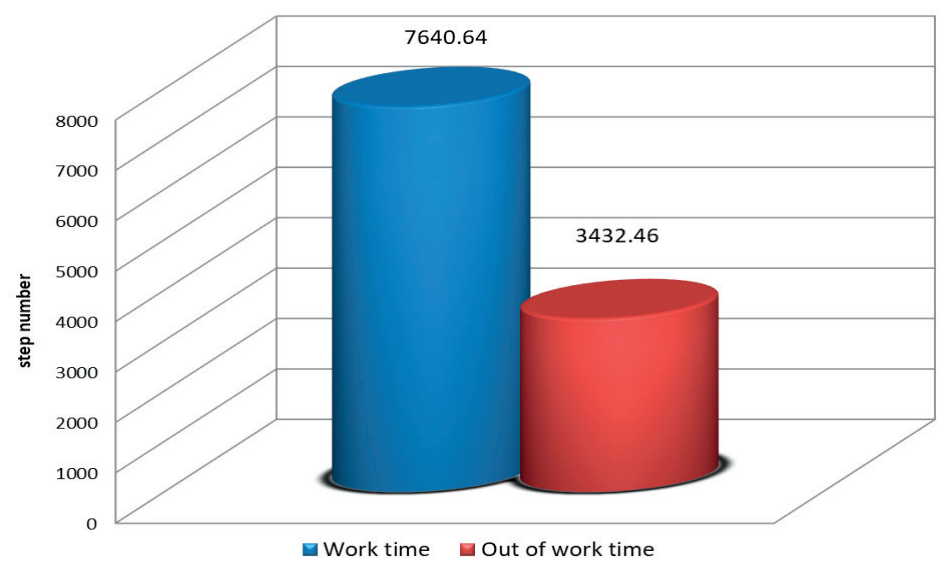

Fig. 9: Average step count during working hours vs off-duty time

The average step count during working hours, as shown in the graph in Figure 9, has been more than double in comparison to off-duty time with the difference being statistically significant $(\mathrm{p}=0.000)$.

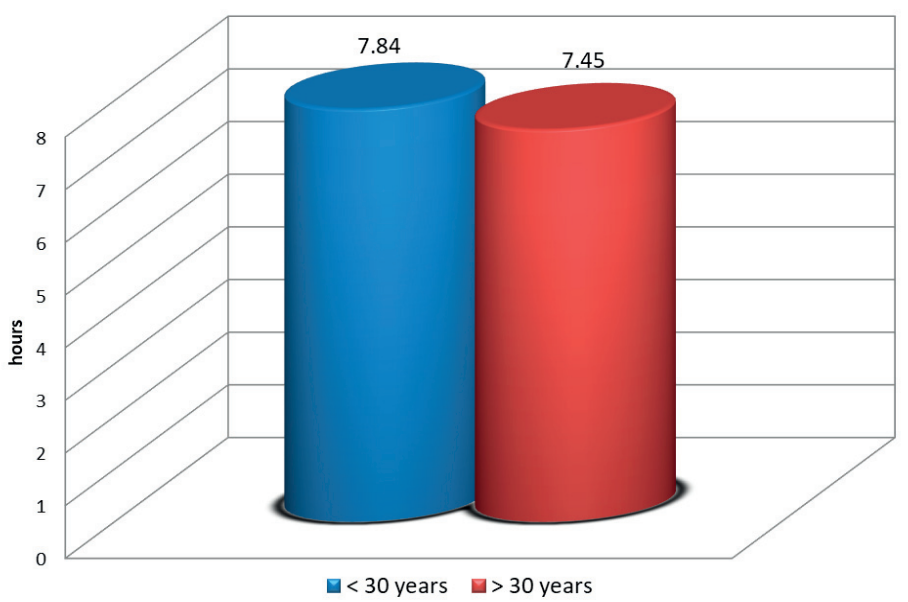

Fig. 10: Average weekly physical activity [hours], calculated for one day, in relation to age

The value of average weekly physical activity [hours], shown in Figure 10, is higher for the ages $\leq 30$ years in comparison to ages $>30$ years, but the difference is not statistically significant $(\mathrm{p}=0.442)$. 


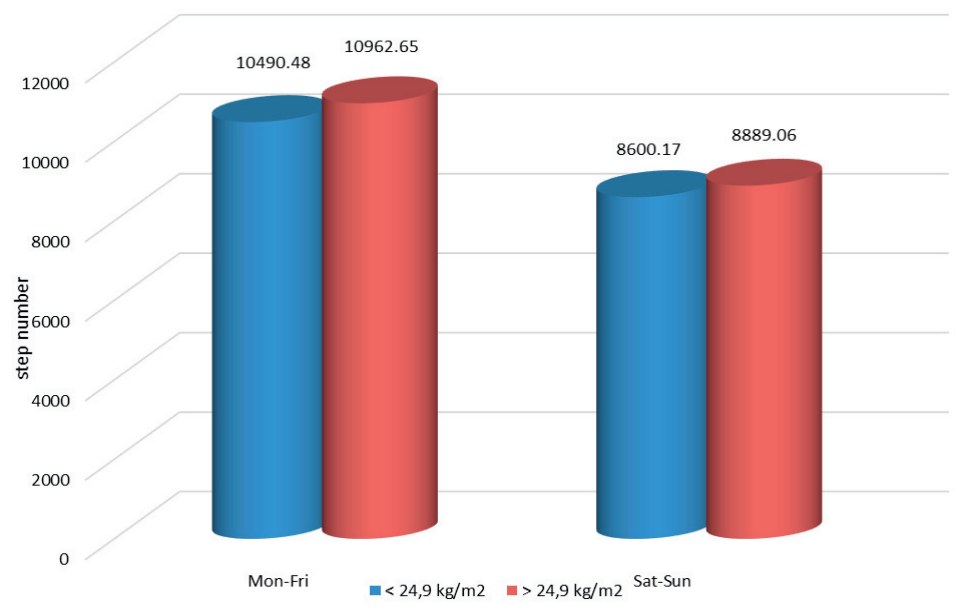

Fig. 11: Average step count calculated for one day

The graph in Figure 11 shows the comparison of number of steps during the weekdays and on weekends in relation to BMI. Individuals with a BMI value $\geq 24.9 \mathrm{~kg} / \mathrm{m}^{2}$ have had a higher step count both during weekdays and weekends in comparison to individuals with values $<24.9 \mathrm{~kg} / \mathrm{m}^{2}$, namely a step count value higher by 536.4 during the week and by 289 during the weekend. Yet this difference is not statistically significant $(\mathrm{p}=0.562)$.

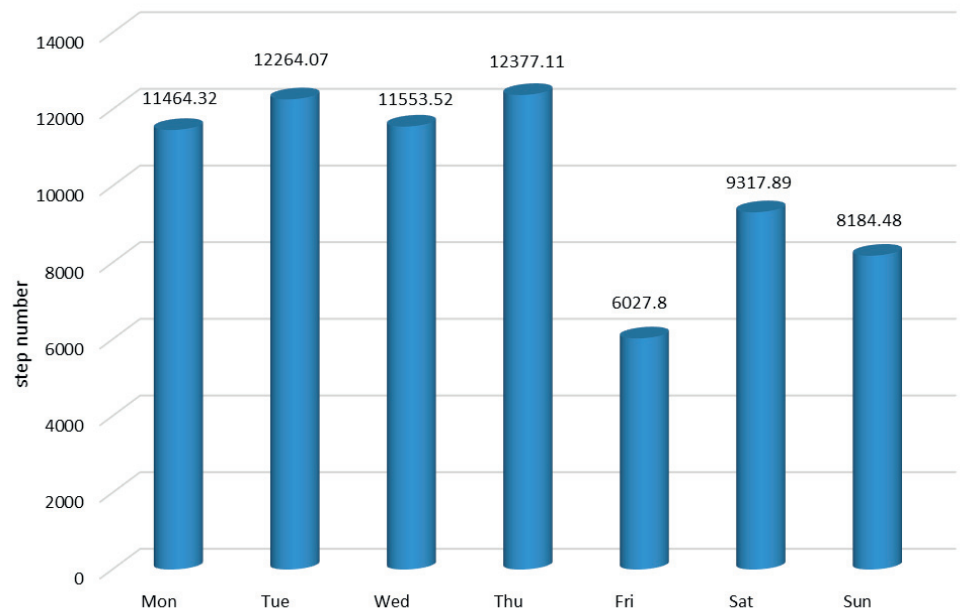

Fig. 12: Average count of steps, leaps and position changes during days of the week

It is evident from the graph shown in Figure 12 that the minimal number of steps, leaps and position changes has been measured on Fridays. The reason behind this is possibly the fact that the working hours on Fridays are 2.5 hours shorter than on the other days (working hours on Mondays to Thursdays being 8.5 hours, on Fridays 6 hours). A more probable reason is that only a smaller part of the monitored individuals is conducting physical training on Fridays. 


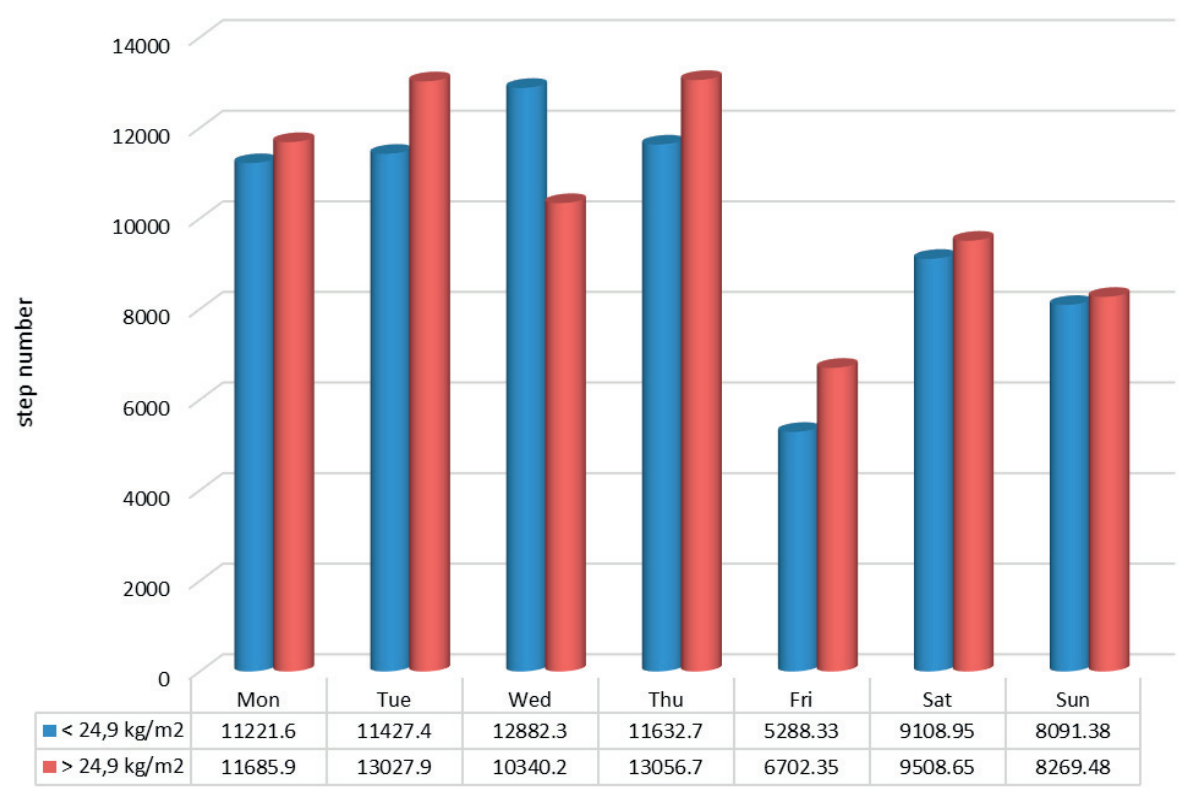

Fig. 13: Average step count during the days of the week in relation to BMI

The graph in Figure 13 shows the of average step count during separate days of the week in comparison to the individuals BMI. The only day during which the individuals with BMI value < $24.9 \mathrm{~kg} / \mathrm{m} 2$ had better results in average step count, with a difference of 2542 steps, is Wednesday. On other days of the week individuals with BMI values $\geq 24.9 \mathrm{~kg} / \mathrm{m} 2$ had the better results, with the differences in step counts being 464.3 on Monday, 1600.53 on Tuesday, 1424 on Thursday, 1414 son Friday, 399.7 on Saturday and 178 on Sunday.

\section{DISCUSSION}

The average value of weekly physical activity (PA) calculated for one day has been 7.66 hours. In contrast the average value of weekly inactivity calculated for one day has been 5.89 hours. It is apparent from these values that the monitored individuals have been active for a larger part of the day than inactive. 9 individuals out of the monitored group have been physically active for more than 9 hours a day on average and 3 physically inactive for more than 9 hours a day on average. The highest average value of physical activity has been 11.71 hours and the highest value of average inactivity 10.28 hours. The measured values of physical activity and inactivity can be compared, for example, with the military police of the Armed Forces of The Czech Republic, where physical activity was measured 7.74 hours and physical inactivity 5.59 hours (Štégner, 2014). The average time of measurement for one day has been 13.55 hours. This value influences the accuracy of the measurement since the measurement accuracy is proportional to the time-span of the measurement. It is evident that the monitored individuals have been wearing the device for most of the day.

The average BMI value of the group - 25.5 can be compared with Czech Combat troops - 26.0 or Logistic troops - 24.9 (Soumar \& Oberman, 2010). The World Health Organisation recommendation for health sustainment (WHO, 2012) is to conduct medium intensity activity (3-6 METs) for at least 2.5 hours a week or conduct intensive physical activity for at least 75 minutes a week or to combine these variants appropriately. 
The average time of medium intensity activity (3-6 METs) has been 34.6 min, which corresponds to 4 hours a week. It is evident that the monitored personnel have met the WHO recommendations. Out of the whole monitored group $79 \%$ have met the parameters of the WHO recommendation for health sustainment. Average daily intensive activity (6-9 METs) has been conducted for a time of 5.59 minutes. The WHO recommendation of 75 minutes of intensive physical activity a week has been met by 10 of the monitored individuals. The highest value of the average intensity activity has been 19.43 minutes, thereby none of the monitored group has reached the value of 22 minutes a day.

Average daily intensity (9-12 METs) has been sustained for the time of 1.91 minutes, where 2 individuals reached a value over 15 minutes ( 16.86 and 21.14 minutes). 5 of the individuals have sustained an average daily activity of values higher than 12 METs for a time larger than 6 minutes with the longest time being 9.86 minutes.

The energy used for physical labour is expressed by a characteristic related to the active energy output. The average daily active energy output has been $622.02 \mathrm{kcal}$. Average active energy output per hour has been $49.05 \mathrm{kcal} /$ hour. Average active relative energy output per day has been $0,59 \mathrm{kcal} / \mathrm{kg} /$ hour. This value can be compared, for example, with Sigmund et al. (2005) who is recommending a value of average daily active relative energy output of $6.5-10 \mathrm{kcal} / \mathrm{kg} /$ day, which corresponds to $0.27-0.42 \mathrm{kcal} / \mathrm{kg} /$ hour. From these values we can deduce that the measured average active relative energy output is more than sufficient.

Overall energy output values are composed of the sum of the active energy output and of the basal metabolism. The average value of the overall energy output per 24 hours has been 2654.59 kcal. This can be compared for example with Bunc (1990) who is recommending a value of 2200 $\mathrm{kcal} /$ day for physical fitness sustainment and a minimum value of $2600 \mathrm{kcal} /$ day for physical fitness improvement or to members of the American army at CBRN (chemical, biological, radiological and nuclear) training - $2420 \mathrm{kcal}$ (Goetz et al., 2011), members of fire brigade - $2585 \pm 406$ kcal (Heil, 2002), Malaysian soldiers - 2886 kcal (Ismail et al., 1996) or the American special operation forces - $3903.8 \mathrm{kcal}$ (Margolis et al., 2014).

The values for physical fitness sustainment ( $2200 \mathrm{kcal} /$ day $)$ have been achieved by 38 individuals of the monitored group, the values for physical fitness improvement ( $2600 \mathrm{kcal} /$ day $)$ by 29 .

Uniaxial accelerometers provide acceleration measurement only in one direction, usually the vertical direction. The principle of using accelerometers for approximating physical activity is based on the relationship of acceleration being directly proportionate to muscle work and thus related to energy output (Montoye et al., 1996). They also measure both the intensity of movement as well as the movement itself. However not all physical activity is manifested in the form of acceleration or deceleration.

The average daily step count ( $10186 \pm 2738$ steps) during a week of continuous field training in a military training area can be categorized as "regular medium intensity movement without competitive sport activity" and military personnel meet the recommended daily step count (Máček et al., 2010).

\section{CONCLUSION}

Although the values of average physical activity, average active relative energy output and recommended average strain intensity have been sufficient, and the healthy recommended average daily step count has been superseded, I recommend to adhere to the appointed time schedules for organised physical training of military personnel during working hours as well as to sustain committing of-duty time to physical activity. Military personnel also have the option to use the facilities and equipment in the garrison - for example the running course, gym, tennis courts, 
squash and foot tennis courts, spinning, climbing facility, massage. Vouchers for downhill skiing, swimming pool and sport centres are available as well.

\section{ACKNOWLEDGMENTS}

Thanks to doc. Mgr. Josef Mitáš, Ph.D. for providing data processing assistance and all people who were a part of the research. The research was supported by the research grant from the GAČR 15-13980S.

\section{References:}

Aadland, E., \& Ylvisåker, E. (2015). Reliability of the Actigraph GT3X+ Accelerometer in Adults under Free-Living Conditions. PLOSONE, 10 (8): e0134606.

Abel, M., G., Hannon, J., C., Sell, K., Lilie, T., Conlin, G., \& Anderson, D. (2008). Validation of the Kenz Lifecorder EX and ActiGraph GT1M accelerometers for walking and running in adults. Applied Nutrition, Physiology and Metabolism, 6, $1155-1164$.

Brage, S., Wedderkoop, N., Franks, P., W., Andersen, L., B., \& Froberg, K. (2003). Reexamination of validity and reliability of the CSA monitor in walking and running. Medicine and Science in Sports and Exercice, 35(8), 1447-1454.

Bunc, V. (1990). Biocybernetic approach to assessing body response to body load. Prague: VÚT UK.

Goetz, V., Yokota, M., Karis, A., J., \&Tharion, W., J. (2011). Energyexpenditure and metabilocheatproductionstorageestiamtes of tacticallawenforcementpersonnelduringchemical, biological, radiological and nuclear (CBRN) training. U.S. Army Research Institute of Environmental Medicine. Natick, MA 01760-5007.

Heil, D., P. (2002). Estimatingenergyexpenditure in wildlandfirefightersusing a physicalactivity monitor. AppliedErgonomics, $33(5), 405-413$.

Chytil, J. (2006). Software ActiPA2006 [Computer Software]. Olomouc: SoftWare Center.

Hamar, D., \& Lipková, J. (2001). Physiology of Physical exercises. Bratislava: Polygraphic center.

Ismail, M., N., Isa, M., \&Janudin, A. (1996). Energyrequirements of Malaysiansoldiers in a base camp. MalaysianJournal of Nutrition, 2, 168-174.

Law no. 221/1999 Collection.

Máček, M., Máčková, J., \& Smolíková, L. (2010). Number of steps as a fitness indicator. Medicina Sportiva Bohemica et Slovaca, 19, 115-121.

Margolis, L., M., Crombie, A., P., McClung, H., L., McGraw, S., M., Rood, J., C., Montain, S., J., \& Young, A., J. (2014). EnergyRequirements of US ArmySpecialOperationForcesDuringMilitaryTraining. Nutrients, 6(5), 1945-1955.

Ministry of Defence of the Czech Republic. (2001). Basic Order of the Armed Forces of the Czech Republic. Prague.

Ministry of Defence of the Czech Republic. (2005). Prog-1-3 (Unit preparation program). Vyškov.

Ministry of Defence of the Czech Republic. (2011). Decree of the Ministry of Defence nr. 12/2011. Prague.

Mitáš, J., Sigmund, E., Frömel, K., Pelclová, J., \& Chmelík, F. (2007). Data processing and a feedback from the monitoring of Physical aktivity using accelerometer Actigraph in the software ACTIPA2006. Czech kinanthropology, 11(4), 40-48.

Montoye, H. J., Kemper, H. C. G., Saris, W. H. M., \& Washburn, R. A. (1996). Measuring Physical activity and energy expenditure. Human Kinetics, 72-79.

Powers, S. K., \& Howley, E. T. (1997). Exercise Physiology: Theory and Application to Fitness and Performance. Madison: Brown and Benchmark.

Rothney, M., P., Schaefer, E., V., Neumann, M., M., Choi, L., \& Chen, K., Y. (2008). Validity of Physical Activity Intensity Predictions by ActiGraph, Actical and RT3 Accelerometers. Obesity (Silver Spring), 16(8), 1946-1952.

Sigmund, E., Frömel, K., \& Neuls, F. (2005). Physical activity of youth: Evaluation guidelines from the viewpoint of health support. Acta Gymnica, 2, 59-68.

Soumar, L., \& Oberman, Č. (2010). Long-Term Monitoring of Actual Health Status Parameters of Czech Population with Emphasis on ACR Servicemen. Czech Military revue, XIX, 174-189.

Štégner, K. (2014). Physical activity of military policemen in the weekly cycle. Diploma thesis, Palacký University Olomouc, Olomouc.

Vilikus, Z., Brandejský, P., \& Novotný, V. (2004). Physical medicine. Prague: Karolinum.

WHO (2012). Global Strategy on Diet, Physical Activity and Health. Physical Activity and Adults. Retrieved from World Wide Web: http://www.who.int/dietphysicalactivity/factsheet_adults/en/index.html.

\section{Contact information:}

zemanekj@volny.cz 